BV acceptance of this article, the publisher or recipient acknowledges the U.S. Government's right to retain a nonexclusive, rovalty-free license in and to anv copyright covering the article.
This report was prepared as an account of work sponsored by an agency of the United State Government. Neither the United States Government nor any agency thereof, nor any of their employees, makes zny warranty, express or implied, or assumes any legal liability or responsibility for the accuracy, completeness, or usefulness of any information, apparatus, product, or process disclosed, or represents that its use would not infringe privately owned rights. Reference herein to any specific commercial product, process, or service by trade name, trademark, manufacturer; or otherwise does not necessarily constitute or imply its endorsement, recommendation, or favoring by the United States Government or any agency thereof. The views and opinions of authors expressed herein do not necessarily state or reflect those of the United States Government or any agency thereof.

\author{
CONF-830776--1 \\ TRANSIENT HEAT TRANSEER IN SUPERFLUID HELIUM, PART II*
}

CONF-830776--i

Fusion Energy Division

Oak Ridge National Laboratory

DE83 015767

\title{
INTRODUCTION
}

Three classical problems associated with the ordinary diffusion equation concern the temperature in (1) a hal.f-space with clamped heat flux at the free face, (2) a half-space with clamped temperature at the free face, and (3) an infinite medium with a pulsed plane heat source. These problems are also important for the nonlinear diffusion equation based on the Gorter-Mellink relation, which describes heat transport in superfluid helium. A similarity solution to problem (1), the clamped-flux problem, has already been found and compared, with good agreement, with experimental data of van Sciver. ${ }^{2}$ [A similarity solution is one in which the profiles of temperature rise $\Delta T$ versus distance $Z$ at different times $t$ can be obtained from one another by suitable (different) stretching of the temperature and distance axes.] In this paper, I give similarity solutions in analytic form to problems (2) and (3), the clampedtemperature and pulsed-source problems.

Both of the latter problems have been studied experimentally and both have practical uses. According to seyfert et al., 3 the clamped-temperature problem describes recovery from burnout (phase transition to He-I at the heated surface) in the presence of steady heating. The pulsed-source problem, studied experimentally by Lottin and van Sciver, ${ }^{4}$ describes the late stages of disappearance

\footnotetext{
*Research sponsored by the Office of Fusion Energy, U.S. Department of Energy, under contract W-7405-eng-26 with the union Carbide Corporation.
} 
of a zone of warm He-II in a long channel. The application of the similarity solutions to these problems and their comparison with the experimental data are explained below.

\section{SIMILARITY SOLUTIONS}

The Gorter-Mellink relation states that in superfluid helium

$$
\vec{q}=-K(\nabla T)^{1 / 3}
$$

where $q$ is the heat flux $\left(w \cdot \mathrm{m}^{-2}\right), \nabla T$ is the temperature gradient $\left(\mathrm{K} \cdot \mathrm{m}^{-1}\right)$, and $\mathrm{K}$ is a thermal conductance parameter $\left(\mathrm{W} \cdot \mathrm{m}^{-5 / 3} \cdot \mathrm{K}^{-1 / 3}\right)$. Combined with the equation of energy conservation,

$$
\nabla \cdot \vec{q}+s \frac{\partial T}{\partial t}=0
$$

where $s$ is the volumetric heat capacity $\left(\mathrm{J} \cdot \mathrm{m}^{-3} \cdot \mathrm{K}^{-1}\right)$, eq. (I) gives

$$
\nabla \cdot\left[K(\nabla T)^{1 / 3}\right]=s \frac{\partial T}{\partial t}
$$

In slab geometry with constant thermophysical properties, eq. (3) becomes

$$
K \frac{\partial}{\partial z}\left(\frac{\partial T}{\partial z}\right)^{1 / 3}=s \frac{\partial T}{\partial t}
$$

A solution of eq. (4) obeying the boundary conditions

$$
\begin{aligned}
& T(0, t)=T_{0}<T_{\lambda} \\
& T(z, 0)=T_{b} \\
& T(\infty, t)=T_{b}
\end{aligned}
$$

of the clamped-temperature problem is

$$
\begin{aligned}
\frac{T-T_{b}}{T_{0}-T_{b}} & =1-\frac{x}{\left(\frac{8}{3 \sqrt{3}}+x^{2}\right)^{1 / 2}} \\
x & =\left(T_{o}-T_{b}\right)^{1 / 2} z(S / K t)^{3 / 4}
\end{aligned}
$$

The reader can verify that $(6 a, b)$ is a solution by substitution. (How to discover such a solution, rather than to verify it once it is known, is described partially in Ref. 1 and in exhaustive detail in Ref. 5.) 
A solution of eq. (4) obeying the boundary conditions

$$
\begin{aligned}
& \int_{-\infty}^{+\infty} S\left(T-T_{b}\right) d z=Q \quad \text { for all } t \\
& T(z, 0)=T_{b} \\
& T(\infty, t)=T_{b}
\end{aligned}
$$

of the pulsed-source problem is

$$
\begin{aligned}
& \frac{(K t / S)^{3 / 2}\left(T-T_{b}\right)}{(Q / S)^{2}}=\frac{4 / 3 \sqrt{3}}{\left(x^{4}+b^{4}\right)^{1 / 2}} \\
& x=2(g / S)(K t / S)^{-3 / 2} \\
& b=2[\Gamma(1 / 4)]^{2} / 3 \sqrt{3 \pi}=2.855
\end{aligned}
$$

This solution, too, can be verified by substitution.

THE PULSED-SOURCE PROBLEM

Shown in the inset in Fig. I are Lottin and van Sciver's temperature profiles at various times after the heat pulse. According to eq. (8), all of these profiles should collapse to a single curve if we use the similarity variables $z / t^{3 / 2}$ and $\left(T-T_{b}\right) t^{3 / 2}$. Shown in the main part of Fig. 1 are the points from the inset replotted in terms of the similarity variables. They do, as expected, define a single curve, which should be given by eq. (8a). Since the temperature rise is only tenths of a degree or less after just a few hundred milliseconds, it is a satisfactory approximation to give $\mathrm{K}$ and $\mathrm{S}$ their values at $\mathrm{T}_{\mathrm{b}}=1.8 \mathrm{~K}$, namely, $\mathrm{K}=10.4 \mathrm{~W} \cdot \mathrm{cm}^{-5 / 3} \cdot \mathrm{K}^{-1 / 3}$ and $S=0.410 \mathrm{~J} \cdot \mathrm{cm}^{-3} \cdot \mathrm{K}^{-1} \cdot 1$ The curve .in $\mathrm{Fig} .1$ has been calculated with eq. (8a) using these values. Agreement is excellent, reinforcing the conclusion of ref. 1 that the Gorter-Mellink relation gives an excellent description of heat transport in superfluid helium.

DISAPPEARANCE OF A WARM ZONE IN A LONG CHANNEL

As mentioned in the introduction, eq. (8) describes the late stages in the disappearance of a warm He-II zone in a long channel, such as mi ynt be found in a cable-in-conduit conductor. 6 In the early stages, the temperature profiles depend on the extent and duration of the heat deposition, but a long time after the heat is deposited, the temperature profiles approach those of an instantaneous 


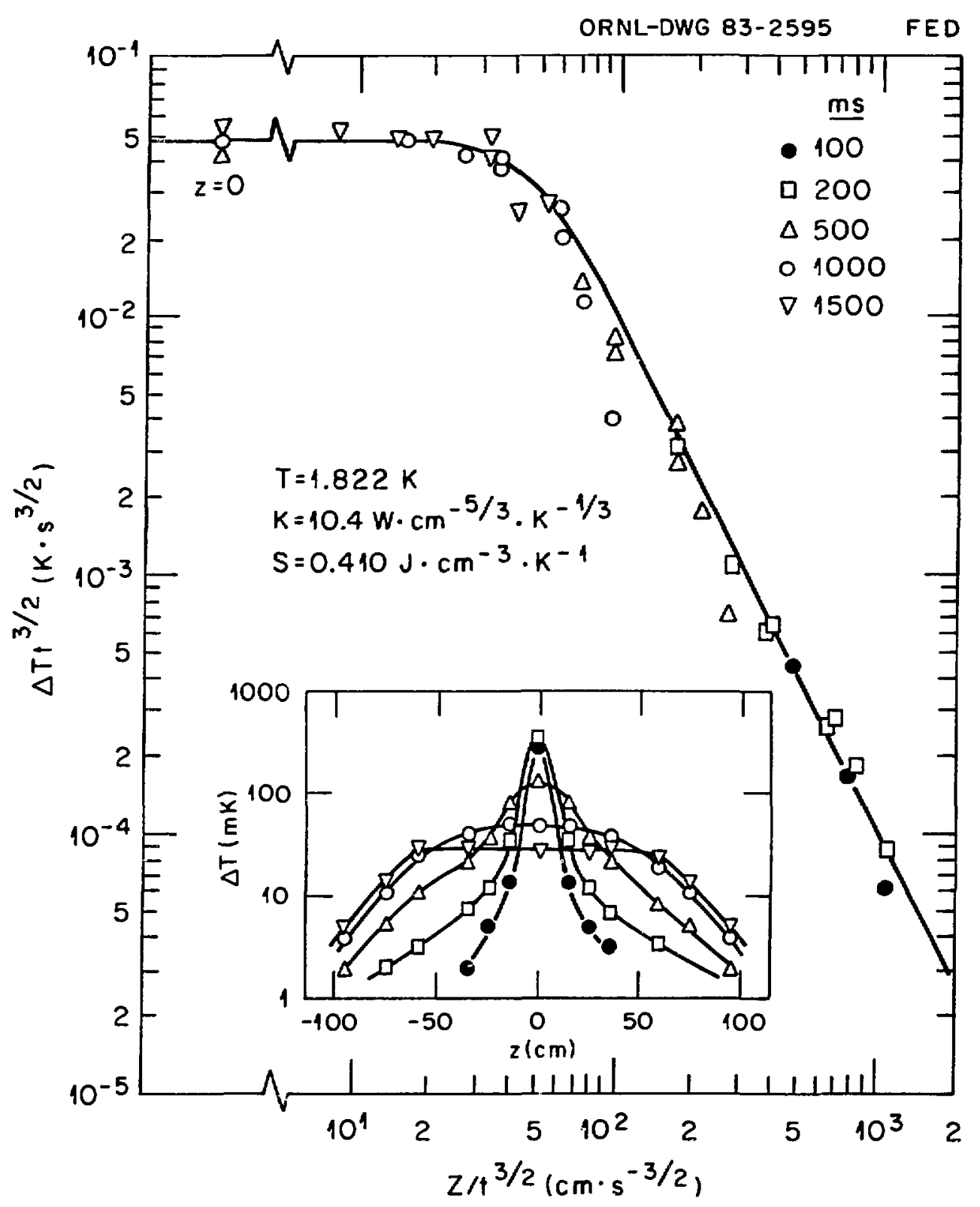

Fig. 1. Temperature distribution in a 6-mm-diam He-II channel after a $0.92-J$ pulse. Experimental data from Lottin and van Sciver (Ref, 4). 
plane source. If the initial temperature of the warm zone lof length $L)$ is $T_{\lambda}$, then the central $(Z=0)$ temperature rise reaches the value $\Delta T \ll T_{\lambda}-T_{b}$ in a time $t$ given by

$$
t=0.207 \frac{s}{k}\left[\frac{\left(T_{\lambda}-T_{b}\right)^{2} L^{2}}{\Delta T}\right]^{2 / 3}
$$

For $T_{b}=1.8 \mathrm{~K}$ and $\mathrm{L}=1 \mathrm{~m}, \Delta \mathrm{T}$ reaches $0.1\left(\mathrm{~T}_{\lambda}-\mathrm{T}_{\mathrm{b}}\right)=37 \mathrm{mK}$ in about $9.1 \mathrm{~s}$, while for $\mathrm{L}=10 \mathrm{~m}, t$ is about $3.3 \mathrm{~min}$.

It is neither impossible nor even unlikely for the currentsharing threshold temperature to be larger than the temperature $T_{\lambda}$ of phase transition. So after reccvery from a transient normality the helium opposite the normal zone may be warmed to a temperature $\mathrm{T}_{1}>\mathrm{T}_{\lambda}$. In this case, the rate-limiting step in recovery may be longitudinal heat conduction in the warm, He-I-filled region. While this problem is strictly speaking not within the announced compass of this paper, it is too important not to be dealt with here. Figure 2 shows (a) a sketch of the physical arrangement and (b) a sketch of the assumed temperature distribution. In the He-II region we assume a linear temperature profile between $T_{\lambda}$ at the

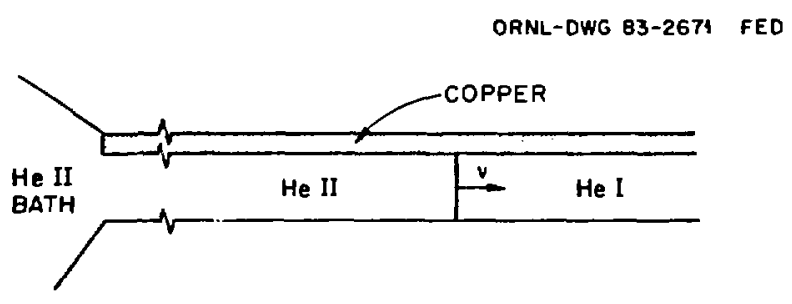

(a)

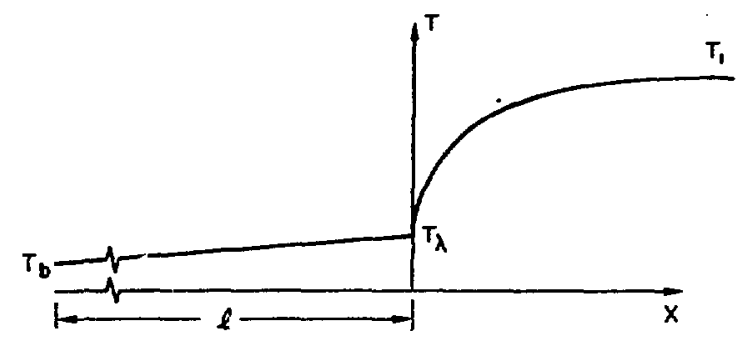

(b)

Fig. 2. Sketch of (a) the conceptual physical arrangement and (b) the assumed temperature profile used in calculating the velocity of a He-I-He-II front in a long channel. 
He-II-He-I interface and $T_{b}$ at the open end of the channel. In the He-I region, the temperature obeys the diffusion equation

$$
A_{H e} S_{H e I} \frac{\partial T}{\partial t}=A_{C u} k_{C u} \frac{\partial^{2} T}{\partial x^{2}}
$$

where $S_{H e I}$ is the volumetric heat capacity of the He-I region, $A_{H e}$ and ${ }^{A}{ }_{C u}$ are the cross-sectional areas of heliun and copper in the unit cell composen of channel and conductor, and $\mathrm{k}_{\mathrm{Cu}}$ is the thermal conductivity of copper. Equation (10) is based on the simplifying assumption that the main contribution to the specific heat is made by the helium and the main contribution to the longitudinal conduction is made by the copper: The related boundary conditions are

$$
\begin{aligned}
& T=T_{\lambda} \text { at the He-I-He-II front } \\
& T=T_{1} \text { Iiar to the right of the front } \\
& k_{C u}{ }^{A} \mathrm{Cu}\left(\frac{\partial T}{\partial z}\right)=k\left(\frac{T_{\lambda}-T_{b}}{\ell}\right)^{1 / 3}{ }_{H e} \text { at the front }
\end{aligned}
$$

A traveling wave solution $T(z-v t)$ must obey the ordinary differential equation

$$
{ }_{\mathrm{He}} \mathrm{S}_{\mathrm{HeI}} \dot{\mathbf{T} v}+{ }_{\mathrm{Cu}{ }^{\mathrm{k}} \mathrm{Cu}} \ddot{\mathbf{T}}=0
$$

where the dots denote differentiation of $T$ with respect to its single argument. If we integrate once and use boundary conditions (1la) and (Ilc) at the front, we get

$$
A_{\mathrm{He}} S_{\mathrm{HeI}} \mathrm{Tv}+{ }_{\mathrm{Cu}}{ }^{k} \dot{\mathrm{T}}=\mathrm{K}\left(\frac{\mathrm{T}_{\lambda}-\mathrm{T}_{\mathrm{b}}}{\ell}\right)^{1 / 3} \mathrm{~A}_{\mathrm{He}}+{ }_{\mathrm{He}} \mathrm{S}_{\mathrm{HeI}} \mathrm{vT}{ }_{\lambda}
$$

Far to the right of the front, $T=T_{1}$ and $\dot{T}=0$. Then from (13) we find

$$
v=\frac{k\left(\frac{T_{\lambda}-T_{b}}{l}\right)^{1 / 3}}{S_{H e I}\left(T_{1}-T_{\lambda}\right)}
$$

which, interestingly, is independent of the cross-sectional areas of the helium and copper as well as of the thermal conductivity of the copper.

If a warm zone witii $T_{1}-T_{\lambda}=2 \mathrm{~K}$ is created in the center of a 100-m-long, He-II-filled $\left(T_{b}=1.8 \mathrm{~K}\right)$, cable-in-conduit conductor, 
then $\mathrm{K}\left[\left(\mathrm{T}_{\lambda}-\mathrm{T}_{\mathrm{b}}\right) / \ell\right]^{1 / 3}=0.44 \mathrm{~W} \cdot \mathrm{cm}^{-2}$. Taking $\mathrm{S}_{\mathrm{HeI}}=0.35 \mathrm{~J} \cdot \mathrm{cm}^{-3} \cdot \mathrm{K}^{-1}$, we find $v^{\prime}=0.62 \mathrm{~cm}^{-1} \mathrm{~s}^{-1}$. This is a rather low velocity and a $1-\mathrm{m}-$ long warm zone would take some 2.7 minutes to disappear, while a 10-mlong zone would require nearly half an hour.

RECOVERY FROM BURNOUT: SEYFERT'S MODEL

Seyfert et al. ${ }^{3}$ consider a situation (see Fig. 3) in which the conductor is normalized by a heat pulse per unit face area E, following which it produces a steady power per unit face area $q_{p}$ (postheating). They are interested in the maximum value of $E$ that permits recovery of the superconducting state for a given $q_{p}$.

The model of recovery from burnout proposed by seyfert et al. is best described in their own words: "At the onset of burnout, formation of the thermal barrier starts. The He-II near the heated surface experiences a phase transition. A He-II-He-I interface appears which has its temperature locked at $T_{\lambda}$. . . We assumed that this barrier had a negligible thickness and that it only affected heat transport in He-II by the condition of a constant temperature, i.e., $T=T_{\lambda}$, at the hot end of the channels in our test section." [Italics mine.]

ORNL-DWG B3-2672 FED

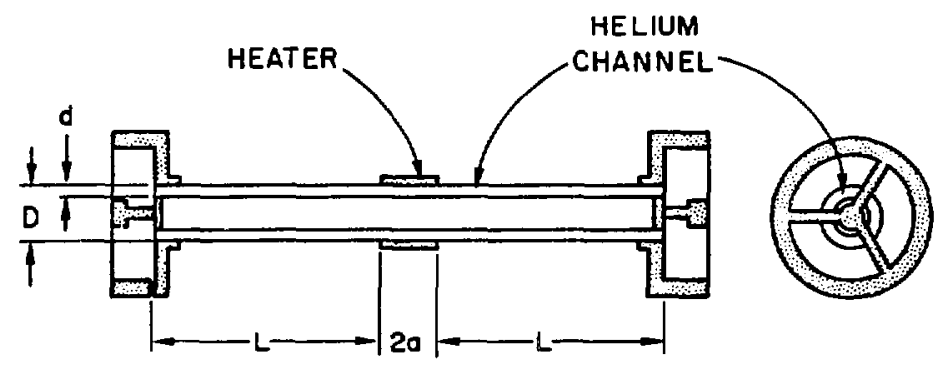

Fig. 3. Sketch by Seyfert et al. ${ }^{3}$ of their experimental channel. Ideally, the heater should be transverse to the channel. The face area to which superficial densities are referred is the cross-sectional area of the channel. Because a circumferential rather than a transverse heater was used, the experimental values of $\mathrm{E}$ obtained by seyfert et al. have an adaied constant included in them that is equal to the enthalpy difference between $T_{\lambda}$ and $T_{b}$ of the helium under the circumferential heater. 
The clamped-temperature similarity solution (6) gives for the instantaneous heat flux into the helium

$$
q=\left(\frac{3 \sqrt{3}}{8}\right)^{1 / 6} \mathrm{~K}^{3 / 4} \mathrm{~s}^{1 / 4}\left(\mathrm{~T}_{\lambda}-\mathrm{T}_{\mathrm{b}}\right)^{1 / 2} \mathrm{t}^{-1 / 4} \equiv \mathrm{At} \mathrm{t}^{-1 / 4}
$$

According to the model of seyfert et al., the maximum heat pulse $E$ still permitting recovery is related to the post-heating power $q_{p}$ by the balance of areas shown in Fig. 4. The algebraic statement of this balance of areas is

$$
E-\int_{0}^{t_{1}} q d t=\int_{t_{1}}^{t_{2}} q d t-q_{p}\left(t_{2}-t_{1}\right)
$$

or

$$
E=\int_{0}^{t_{2}} q d t-q_{p}\left(t_{2}-t_{1}\right)
$$

Substituting (15) into (16) and noting that $t_{2}>t_{1}$ and that $q_{p}=A t_{2}-1 / 4$, we find

$$
E=\frac{1}{3} q_{p} t_{2}
$$

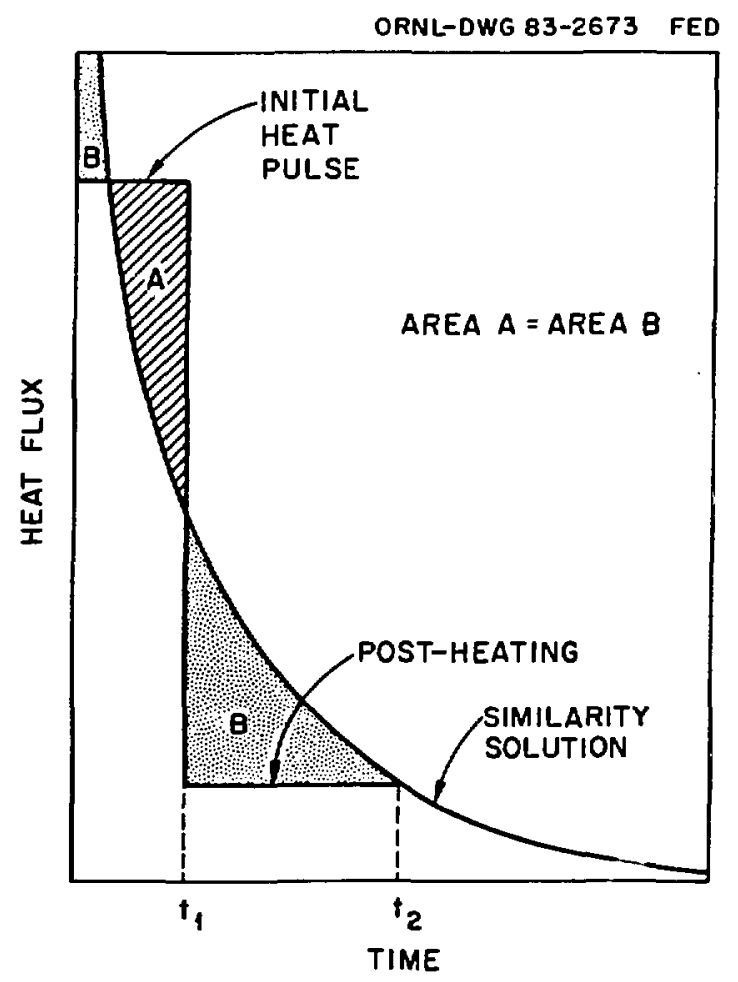

Fig. 4. Balance of areas determining the relation between the initial heat pulse $E$ and the postheating power $q_{p}$, according to seyfert et al. $3^{p}$ 
From (15) we find

$$
t_{2}=\frac{3}{4} K^{3} s\left(T_{\lambda}-T_{b}\right)^{2} q_{p}-4
$$

so that

$$
E=\frac{1}{4} k^{3} S\left(T_{\lambda}-T_{b}\right)^{2} q_{p}^{-3}
$$

COMPARISON WITH EXPERIMENT

The experimental values of $E$ summarized by Sefyfert et al. in Fig. 11 of Ref. 3 include an added constant that is equal to the enthalpy difference between $T_{\lambda}$ and $T_{b}$ of the helium under the circumferential heater in the center of their experimental channel. When this constant is subtracted from their experimental values of $E$, these values are brought into excellent agreement with the results of their numerical calculations. Consequently, I shall compare eq. (19) with the numerical results.

Figure 5 shows the calculated results of Seyfert et al. replotted on log-log paper. The lines, which have a slope of -3 , fit the points quite well, verifying the dependence of $E$ on $q_{p}$ given

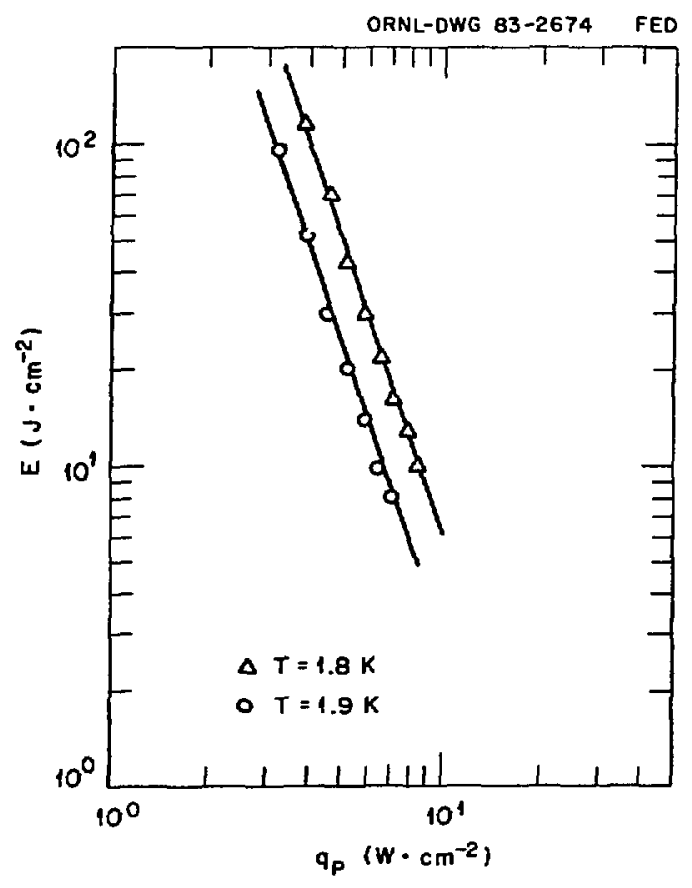

Fig. 5. The maximum allowable heat pulse $E$ as a function of the post-heating power $q_{p}$. The points, taken from the paper of Seyfert et al. ${ }^{3}$ have been corrected for the presence of helium under the circumferential heater. 
by eq. (19). Both lines correspond to $\mathrm{Ks}^{1 / 3}=5.68 \mathrm{~W} \cdot \mathrm{J}^{1 / 3} \cdot \mathrm{cm}^{-8 / 3} \cdot \mathrm{K}^{-2 / 3}$. This value may be compared with the values of $7.73,9.52,10.6$, and $8.62 \mathrm{w} \cdot \mathrm{J}^{1 / 3} \cdot \mathrm{cm}^{-8 / 3} \cdot \mathrm{K}^{-2 / 3}$ at $1.8,1.9,2.0$, and $2.1 \mathrm{~K}$, respectively, obtainable from Table I of Ref. 1. The discrepancy arises because in the clamped-temperature problem, the superfluid elements near the heater have temperatures very close to $T_{\lambda}$. As we approach $T_{\lambda}$ from below, $K$ falls sharply toward zero and $S$ rises (though not to infinity). Presumably, the sharp drop in $K$ near $T_{\lambda}$ is what diminishes the best-fit value of $\mathrm{ks}^{1 / 3}$.

\section{VALIDITY OF THE INFINITE-CHANNEL APPROXIMATION}

The theory of the last two sections applies to an infinitely long channel, whereas the experiments of seyfert et al. refer to a channel of lenth $L=4 \mathrm{~cm}$. When $x \gg 1, T=T_{b}$ according to the similarity solution (6). So if

$$
L \gg\left[\frac{K t}{s\left(T_{\lambda}-T_{b}\right)^{2 / 3}}\right]^{3 / 4}
$$

then $T(L)$ will be close to $T_{b}$ and the infinite-channel approximation should be a valid description of the finite channel. If we substitute from eq. (18) for $t_{2}$, we find

$$
q_{p} \gg\left(\frac{3}{4}\right)^{1 / 4} K\left(\frac{T_{\lambda}-T_{b}}{I}\right)^{1 / 3}
$$

If we take $T_{. \lambda}=2.17 \mathrm{~K}, \mathrm{~T}_{\mathrm{b}}=1.8 \mathrm{~K}, \mathrm{~L}=4 \mathrm{~cm}$, and $\mathrm{K}=5.80 \mathrm{~W} \cdot \mathrm{cm}^{-5 / 3} \cdot \mathrm{K}^{-1 / 3}$ (half the largest value from Ref. 1) we find $q_{p} \gg 2.4 \mathrm{~W} \cdot \mathrm{cm}^{-2}$. For $T_{b}=1.9 \mathrm{~K}, q_{p}$ must be $\gg>2.2 \mathrm{~W} \cdot \mathrm{cm}^{-2}$. So we expect the infinitechannel approximation to be a reasonable one for the points in Fig. 5 .

\section{ACKNOWLEDGMENT}

I would like to acknowledge the contribution of E. Tada of the Japan Atomic Energy Research Institute, who first raised the question of the propagation of a He-II-He-I front in a long channel. I also wish to express my gratitude to Prof. S. W. van Sciver of the University of Wisconsin for sending me the numerical values corresponding to the data points in Fig. 1. 
REFERENCES

1. L. Dresner, Transient heat transfer in superfluid helium, $A d v$. Cryo. Eng. 27:411-419 (1982).

2. S. W. van Sciver, Transient heat transport in He-II, Cryogenics 19:385-392 (1979).

3. P. Seyfert, J. Lafferranderie, and G. claudet, Time-dependent heat transport in subcooled superfluid helium, Cryogenics $22: 401-408$ (1982).

4. J. C. Lottin and S. W. van Sciver, Heat transport mechanisms in a 2.3-meter-long cooling loop containing He-II, in "Proceedings of the Ninth International Cryogenic Engineering Conference, Kobe, Japan, May 11-14, 1982," Butterworth, Guildford, Surrey (1982), pp. 269-272.

5. L. Dresner, "Similarity Solutions of Nonlinear Partial Differential Equations," Pitman Publishing (to be published).

6. M. O. Hoenig, Internally cooled, cabled superconductors cooled with He-II, in "Proceedings of the Saclay Workshop, November 16-19, 1981," International Institute of Refrigeration, Paris, France (1982), Pp. 301-310. 